\title{
APLICACIÓN DEL SOFTWARE TURNITIN Y SU ÍNDICE DE SIMILITUD FINAL, COMO UN INDICADOR DE ORIGINALIDAD EN TESIS DE MEDICINA HUMANA, URP
}

\author{
APPLICATION OF THE TURNITIN SOFTWARE AND ITS INITIAL AND FINAL SIMILARITY, \\ AS AN INDICATOR OF ORIGINALITY IN THESIS OF HUMAN MEDICINE, URP \\ Jhony A. De La Cruz-Vargas',2, Manuel O. Huamán-Guerrero 1,2, Paola Baldeon-Martinez ,2, \\ Claudia Torres-Otárola ${ }^{1,2}$, Consuelo Luna-Muñoz ${ }^{1,2}$ y Lucy E. Correa-López ${ }^{1,2}$
}

\begin{abstract}
RESUMEN
Objetivos: Presentamos un estudio para determinar el índice de similitud final con el uso del software Turnitin ${ }^{\circledR}$ como un indicador de originalidad de las tesis de Medicina Humana de la Universidad Ricardo Palma. Métodos: Se realizó un estudio transversal con enfoque cuantitativo. Se seleccionaron de manera aleatoria 100 tesis realizadas con el Curso Taller de Titulación por Tesis organizado por la Facultad de Medicina Humana-Instituto de Investigación en Ciencias Biomédicas, de la Universidad Ricardo Palma, durante su primera edición año 2015. Las tesis fueron analizadas con el software Turnitin con el fin de detectar el índice de similitud final (ISF). Resultados: De las 100 tesis evaluadas, según sexo de los autores se encontró que el $46 \%$ eran hombres y el 54\% correspondió a mujeres. Los temas preferidos de las tesis, correspondió a las áreas clínicas en un 60\% (Medicina Interna, Salud Publica y Pediatría) y luego las áreas quirúrgicas $40 \%$ (Cirugía y Ginecología). La media obtenida del ISF fue de 8.41. De manera interesante el $65 \%$ de las tesis evaluadas obtuvo un Índice menor a $10 \%$. Solo el $15 \%$ de las tesis correspondió a un ISF grado IV (>15\%). Cuando revisamos las calificaciones de los jurados de tesis, el $90 \%$ correspondió a las tesis calificadas como buenas, muy buenas y sobresalientes. Conclusión: El software Turnitin ${ }^{\circledR}$ es adecuado para reportar el grado de similitud en las tesis de medicina. El índice de similitud final, constituye un indicador de originalidad, y el software puede ser útil para ayudar a detectar los niveles de originalidad y por lo tanto mejorar la integridad académica en el pregrado. El grado de originalidad hallado en las tesis de medicina humana del curso taller de titulación por tesis 2,015 de la Universidad Ricardo Palma es satisfactorio.
\end{abstract}

Palabras clave: Plagio; Tesis; Ética; Integridad académica (fuente: DeCS BIREME).

\begin{abstract}
Objective: We present a study to determine the "Final Index of Similarity" with the use of the Turnitin ${ }^{\circledast}$ software as an indicator of originality on the theses of Human Medicine of the Ricardo Palma University. Methods: A cross-sectional study was conducted with a quantitative approach. A total of 100 theses were randomly selected Performed with the Course Workshop to obtain the title of Medical surgeon, organized by the School of Human Medicine-Biomedical Sciences Research Institute, Ricardo Palma University, during its 1rst edition in 2015. The theses were analyzed with the Turnitin software in order to detect the rate of Similarity (FIS). Results: Of the 100 theses evaluated, according to the authors' gender, there were $46 \%$ men and $54 \%$ women. The preferred subjects of the theses corresponded to the clinical areas in $60 \%$ (Internal Medicine, Public Health and Pediatrics) and then surgical areas $40 \%$ (Surgery and Gynecology). The average obtained from the FIS was 8.41 . Interestingly, $65 \%$ of the theses evaluated had an index of less than $10 \%$. Only $15 \%$ of the theses corresponded to a FIS grade IV (>15\%). When we reviewed the qualifications of the thesis jurors, $90 \%$ corresponded to the theses described as good, very good and outstanding. Conclusions: The Turnitin software is adequate to report the degree of similarity in medical theses. The Final Index of Similarity is an indicator of originality, and the software can be useful to help detect levels of originality and therefore academic integrity in the undergraduate. The degree of originality found in the theses of human medicine of the course workshop in 2015 of the Ricardo Palma University is satisfactory.
\end{abstract}

Key words: Plagiarism; Thesis; Ethics (source: MeSH NLM).

'Instituto de Investigación en Ciencias Biomedicas, Universidad Ricardo Palma.
2 Facultad de Medicina Humana, Universidad Ricardo Palma.

Correspondencia: Prof. Jhony A De La Cruz, Director Adjunto del INICIB, Facultad de Medicina Humana, Edificio1. 2 do Piso. Dirección: Av. Benavides 5440, Surco-Lima, Perú. Universidad Ricardo Palma. Telefóno: 708-0000. Anexo: 6016. Correo: jhony.delacruzv@urp.pe

Citar como: Jhony A. De La Cruz-Vargas, Manuel O. Huamán-Guerrero, Jofre Hidalgo, Paola Baldeon-Martinez, Claudia Torres-Otárola, Consuelo Luna-Muñoz y Lucy E. Correa-López. Aplicación del software turnitin y su índice de simillitud final, como un indicador de originalidad en tesis de medicina humana, URP [Artículo Original]. Rev. Fac. Med. Hum. 2016;16(2):31-38. DOI 10.25176/RFMH.v16.n2.666 


\section{INTRODUCCIÓN}

En la era del conocimiento en la que hoy vivimos, la evaluación de la originalidad y finalmente de la calidad ha trascendido también a la producción científica, representada por las tesis, investigaciones y publicaciones científicas, entre otras ${ }^{1}$.

La tesis de pregrado es un trabajo académico de alto rigor científico considerado como el primer paso serio de los estudiantes universitarios en el campo de la investigación, además de ser requisito indispensable para obtener el título profesional de médico cirujano $^{2}$. La tesis en Medicina vincula el entorno académico universitario y los diversos campos clínicos, proyectándose a una posible aplicación en la sociedad. Ha sido un requisito para el bachillerato y actualmente un paso fundamental para la titulacion ${ }^{3}$.

La evaluación de la calidad de tesis, es una prioridad para las universidades a nivel nacional e internacional. Múltiples autores han comunicado el problema del plagio en pregrado como un problema en crecimiento ${ }^{4,5}$. Diversos softwares denominados antiplagio han sido propuestos: iThenticate, CrossCheck, Ephorus, Approbo, WCopyfind y Turnitin. El plagio se define como "el uso de palabras o ideas de otra persona sin un reconocimiento apropiado" (JU Catalogo, 2006)6. "Robar y transmitir (las ideas o palabras de otro) como propio: usar (la producción ajena) sin acreditar la fuente" y "cometer robo literario: presentar como nueva y original una idea o producto derivado de una fuente existente "(Merriam-Webster Online Dictionary, 2005) ${ }^{7}$. El plagio y otras formas de violaciones a la integridad académica de los estudiantes siempre han sido un dilema para los profesores universitarios y los administradores y alcanzaron proporciones epidémicas durante los años noventa. (Koch, 2000)8.

El software Turnitin; iParadigms LLC, San Francisco, CA, USA ${ }^{9}$, según sus creadores, ayuda al instructor a revisar el texto, cruzándolo con fuentes de internet y trabajos previamente publicados, e identificar posibles coincidencias inadvertidas. En un estudio de revisión de revistas (APAME journals) ${ }^{10}$, se encontró hasta un $75 \%$ de plagio y un $58 \%$ de duplicación de publicaciones. Turnitin puede mejorar la detección de coincidencias alrededor de un 15-20\% ${ }^{10}$.

Carnero A. y col. ${ }^{11}$ publicaron recientemente la experiencia de 7 casos de plagio del 2011-2014, en una maestría en Epidemiologia en Lima, Perú. Depen- diendo de la severidad de los eventos, las penalizaciones incluyeron la desaprobación del curso (6/7) y la separación del programa (3/7). Los estudiantes culpables no se involucraron en más plagio. Los autores afirman que el plagio es un problema grave y extendido, de mala conducta en investigación, y es a menudo descuidado en los países en desarrollo. A pesar de sus implicancias a mediano y largo alcance, el plagio es mal reconocido y discutido en el ámbito académico, y no hay evidencia suficiente en América Latina y en los países en desarrollo para informar sobre la aplicación de estrategias preventivas.

La era digital y el Internet han agravado el tema de las copias, el mayor uso de los sistemas de información por los estudiantes, fuentes electrónicas de información en línea y la facilidad con que se puede descargar y utilizar la información, ha llevado a que el ciberplagio sea un problema actual. (Campbell, Swift, \& Denton, 2000) ${ }^{12}$.

Una de las estrategias que las universidades pueden utilizar como parte de su enfoque al problema de falta de originalidad es desplegar un software electrónico de detección que puede identificar pasajes idénticos de texto en uno o más documentos. Este software puede ser usado para verificar las sospechas de un profesor de que una pieza particular de trabajo es coincidente, o puede ser utilizado para procesar lotes enteros de cursos para permitir la selección de las presentaciones de grupos enteros de estudiantes ${ }^{13}$.

La Universidad Ricardo Palma, dentro de sus políticas institucionales de investigación, ha adquirido la licencia y ha incorporado el uso del software Turnitin, en todas las facultades y unidades académicas de pre y posgrado. En este contexto presentamos un estudio para determinar el índice de similitud final con el uso del software Turnitin como un indicador de originalidad de las tesis de Medicina Humana de la Universidad Ricardo Palma.

\section{MÉTODOS}

Se realizó un estudio no experimental, transversal con enfoque cuantitativo. Se seleccionaron de manera aleatoria 100 tesis realizadas con el "Curso Taller de Titulación por Tesis" organizado por la Facultad de Medicina Humana-Instituto de Investigación en Ciencias Biomédicas, de la Universidad Ricardo Palma, durante su primera edición año 2015. Las tesis fueron analizadas con el software Turnitin con 
el fin de detectar el Índice de similitud inicial (ISI). El objetivo principal de Turnitin es resaltar posibles trabajos no originales de los estudiantes. En resumen, el proceso incluyó: una vez que se cargó el texto en el software, la aplicación proporcionó lo que los fabricantes llaman un "informe de originalidad". El informe sugiere un porcentaje global del texto del alumno que coincide con las fuentes de su base de datos e indica el nivel de coincidencia con un icono de color ( $0=$ azul, 1-24 = verde, 25-49= amarillo, 50-74 = naranja $75-100=$ rojo). Los resultados se generan a través de búsquedas completas de miles de millones de páginas de Internet, millones de trabajos de estudiantes presentados anteriormente en la base de datos de Turnitin y bases de datos comerciales de artículos de revistas y publicaciones (http://www.turnitin.com/static/plagiarism.html).

El software también indicó el porcentaje de texto coincidente para cada fuente nombrada y los presentó como una lista ordenada por cantidad. Con el texto de cada estudiante, el espectador puede hacer clic en una sección adaptada y luego ver el texto "original" sugerido lado a lado para una comparación instantánea entre los dos textos.

El sistema completo de prevención de Turnitin permitió comprobar el trabajo de los estudiantes de manera rápida y efectiva. Luego se hizo una revisión académica de cada resultado: 1. Verificación del listado de coincidencias. 2. Revisión cita por cita 3. Comprobación de referencia o no en el texto. 4. Evaluación de referencia adecuada o no. 5. Obtención del Índice de Similitud Final (ISF) o revisado.

Se generó una base de datos en Excel y posteriormente se realizó un análisis con SPSS 22.0.

\section{RESULTADOS}

De las 100 tesis evaluadas, según sexo de los autores se encontró que el $46 \%$ eran hombres y el $54 \%$ correspondió a mujeres. En relación al número de páginas de las tesis, se obtuvo una media de 73 páginas, con un rango de 34-227 páginas.

El área o tema abordado por las tesis, se ve reflejado en el gráfico 1. El gráfico 2 y 3, muestran los resultados según sexo.

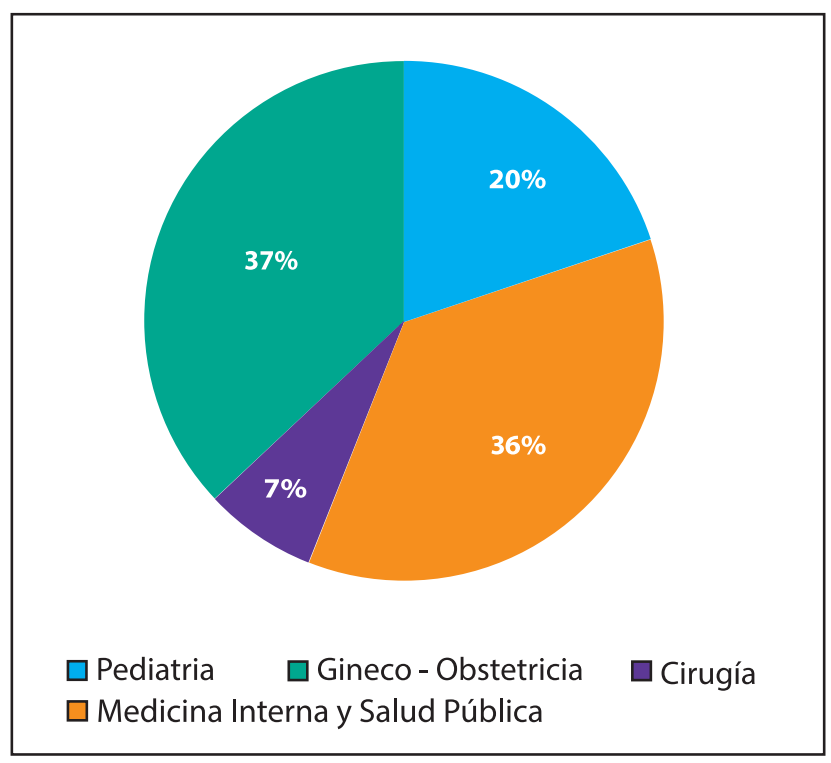

Gráfico 1. Tesis de medicina por áreas en porcentajes.

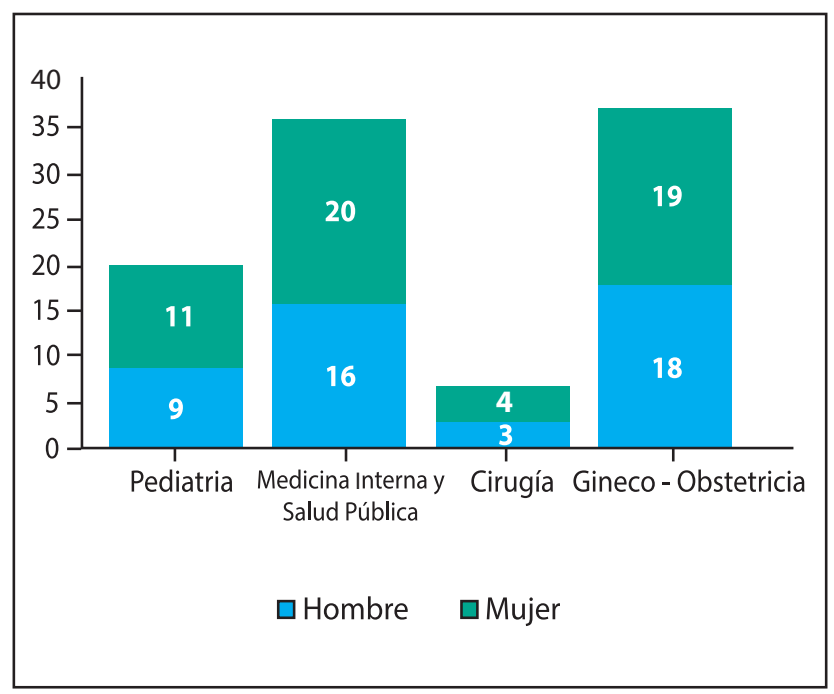

Gráfico 2. Tesis de medicina clasificadas por áreas según sexo.

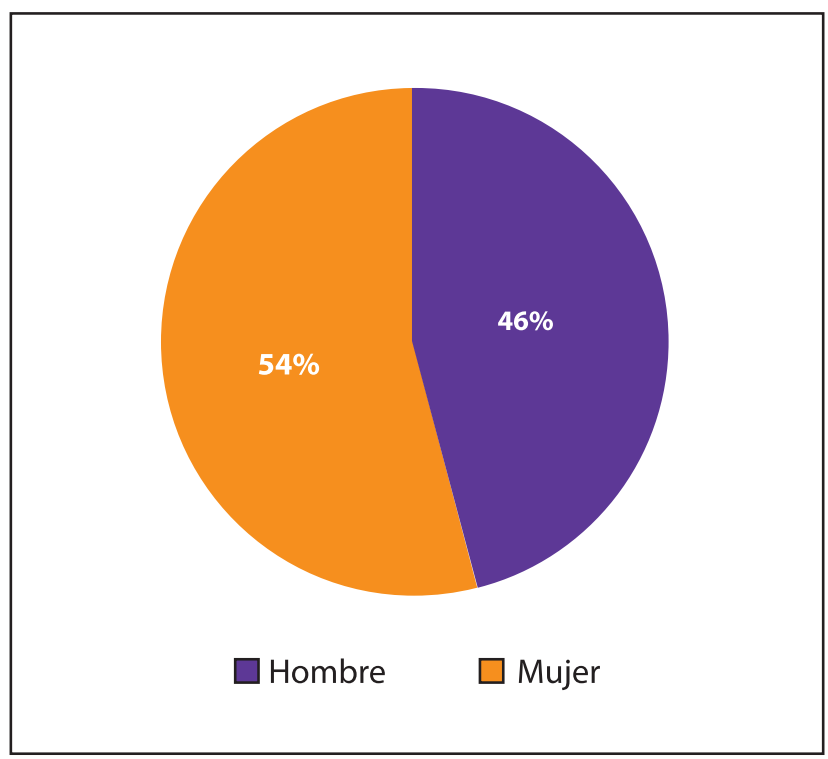

Gráfico 3. Tesis totales según sexo. 
Al utilizar el software Turnitin para cada una de las tesis, se obtuvo en primera instancia el Índice de Similitud Inicial (ISI), dando una media de $55.47 \%$. Se obtuvo el Índice de Similitud Final (ISF), con una media de $8.21 \%$. Luego se estableció cuatro grados de ISF. los grupos establecidos están reflejados en la tabla 1.

Tabla 1. Grupos de índice de similitud final (revisado).

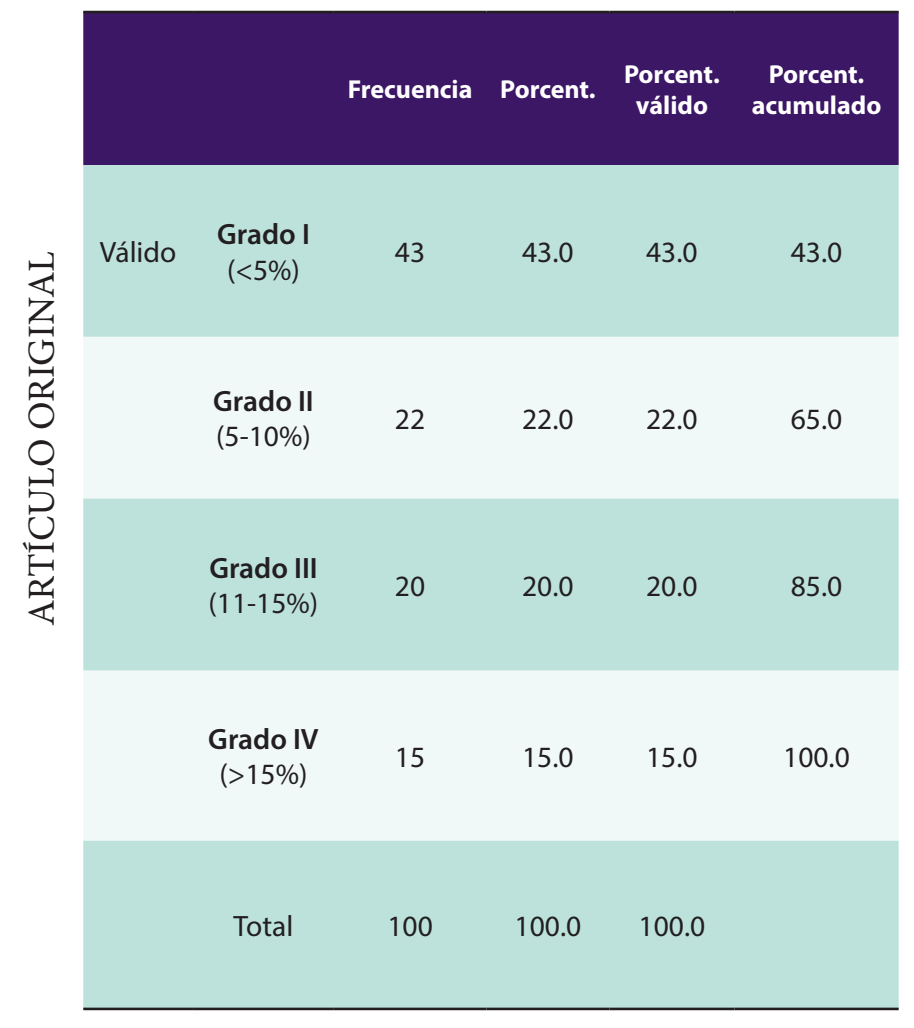

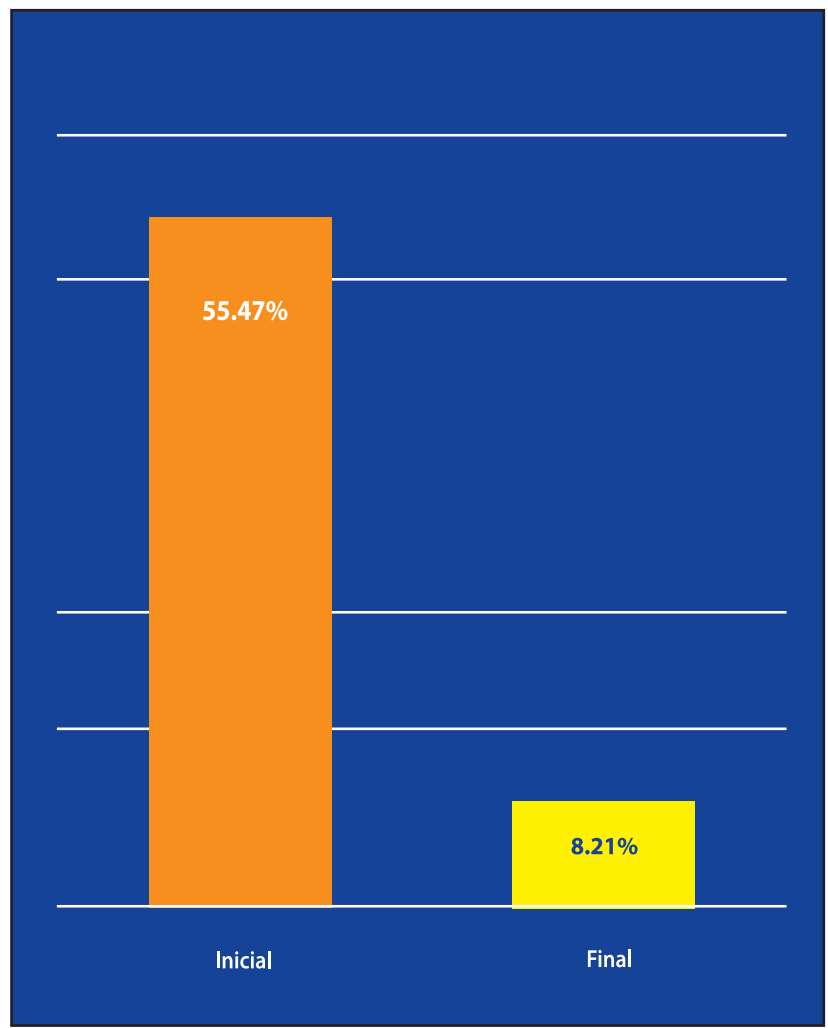

Gráfico 5. Índice de similitud inicial y final con el software Turnitin.

Evaluamos adicionalmente las calificaciones obtenidas por los autores de cada una de las tesis, por sus jurados universitarios correspondientes durante el momento de presentación y sustentación, obteniendo una media de 15.5 con un rango de 13 a 19. Luego se establecieron cuatro grados: Regular, Bueno, Muy Bueno y Sobresaliente.

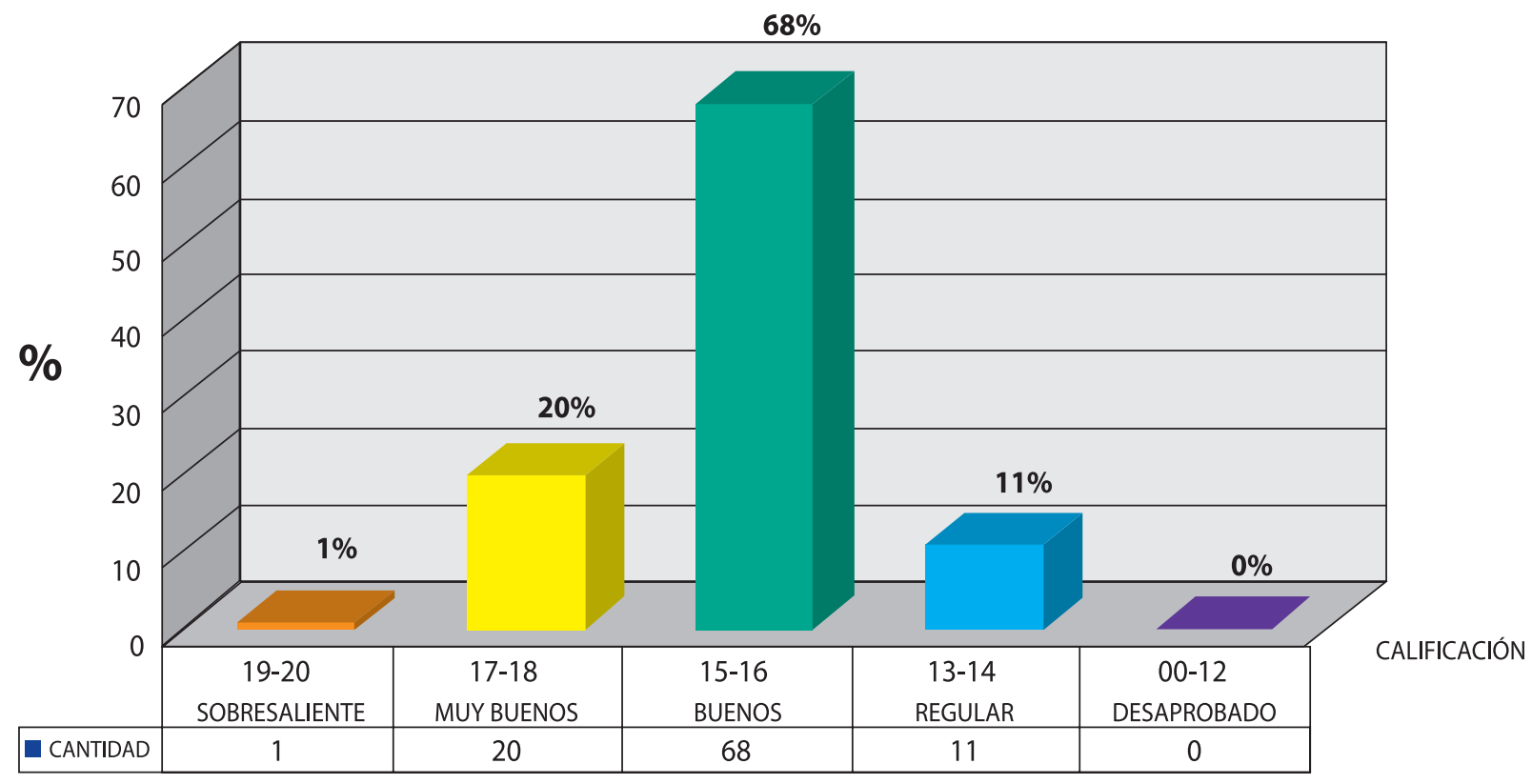

Gráfico 6. Distribución según calificaciones por jurado de tesis. 


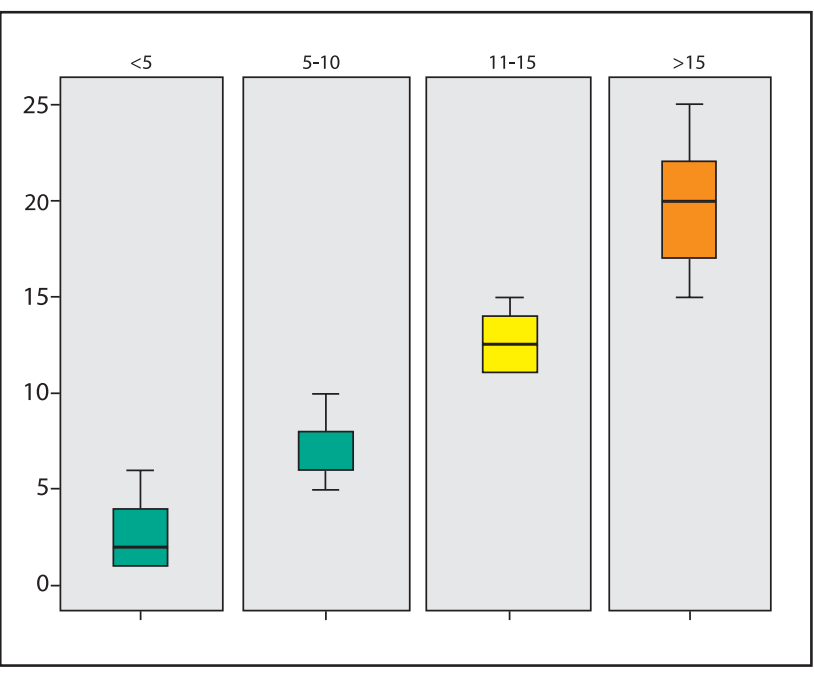

Gráfico 7. Cajas y bigotes según índice de similitud final.

\section{DISCUSIÓN}

Hay una diferencia muy clara entre lo que Turnitin marca como texto coincidente (índice de similitud) y plagio ${ }^{14}$. Turnitin pondrá de relieve cualquier material coincidente en un documento, incluso si se cita y cita correctamente ${ }^{15}$. El hecho de que aparezca como poco original no significa necesariamente que esté plagiado; sólo significa que el material coincide con un texto en las bases de datos de Turnitin. Se deja a los instructores para que ellos revisen el documento y el informe de originalidad para determinar si alguna coincidencia es plagio y hasta qué punto plagio intencional, plagio no intencional, impropio / falta de cita o simplemente coincidencia ${ }^{16}$.

En nuestro estudio, se encuentra una importante presencia femenina en la realización de las 100 tesis evaluadas. Esto va acorde con el "fenómeno rosa" de un aumento significativo de mujeres, observado en los últimos años en las Facultades de Medicina ${ }^{17,18}$.

Por otro lado, los temas preferidos de las tesis, correspondió a las áreas clínicas en un $60 \%$ (medicina interna, salud pública y pediatría) y luego las áreas quirúrgicas 40\% (cirugía y ginecología).

Si bien la media del "Índice de Similitud Inicial" fue de $55.47 \%$, debemos recordar que se aplicó a un grupo de tesis ya sustentadas y sin la oportunidad de haber revisado sus trabajos con Turnitin. De manera muy interesante, cuando las coincidencias fueron revisadas por los instructores, el "Índice de Similitud Final" o Revisado fue de $8.21 \%$. Una de las razones que explican la reducción sustancial en el ISF, es que, al momento de evaluar las tesis, ya habían sido subidas y estaban disponibles online en CYBERTESIS, por lo que al ser evaluadas por Turnitin, aparece el reporte inicial con un gran porcentaje de coincidencias citadas en esas mismas fuentes, mostrándonos el poder de búsqueda del software y la velocidad de encontrar dichas similitudes.

De manera interesante el $65 \%$ de las tesis evaluadas obtuvo un Índice menor a 10\%. Solo el 15\% de las tesis correspondió a un ISF grado IV (>15\%). Cuando revisamos las calificaciones de los jurados de tesis, el $90 \%$ correspondió a las tesis calificadas como buenas, muy buenas y sobresalientes. Cualquier umbral del índice de similitud es arbitrario, el centro de Oxford en el estudio CAVAL (citado por Carroll 2004) utilizó como umbral el 25\% ${ }^{19}$.

En los últimos años ha aumentado la preocupación por el alcance de la originalidad científica en la vida universitaria (Szabo y Underwood, 2004) ${ }^{20}$. La comunidad académica ha respondido con una serie de estrategias diferentes para contrarrestar dicho problema. Algunas de estas estrategias fueron centradas en los estudiantes, por ejemplo, aumentando la concientización de los estudiantes sobre el plagio (Carroll, 2002) ${ }^{21}$, aclarando lo que constituye el plagio o introduciendo códigos de honor (McCabe, 2001)22. Otra respuesta fue la introducción de sistemas cada vez más sofisticados de detección (Frey, 2001)23. Una de las innovaciones más importantes fue la introducción de sistemas electrónicos de detección de similitudes, como Turnitin ${ }^{\circledR}$, una herramienta que permite a los tutores verificar posibles coincidencias y convertirse en una herramienta de presentación y clasificación electrónica más completa.

Dos estudios encontraron una fuerte relación entre la severidad de la deshonestidad académica entre estudiantes y la severidad de la deshonestidad más tarde cuando estos mismos estudiantes estuvieran en el mundo laboral (Nonis \& Swift, 2001, Sims, 1993)24,25. Así, la deshonestidad académica tiene consecuencias de largo alcance más allá del campo de la educación superior. Por lo tanto, es importante explorar el potencial del uso de la tecnología actual para identificar y disuadir posibles copias.

Una de las características esenciales de una tesis o trabajo de investigación, es la originalidad. ¿Pero que es la originalidad? Pablo Raúl Bonorino Ramírez, de la Universidad de Vigo, explica: "La originalidad en un proyecto de investigación puede significar muchas cosas. Un error muy común en investigadores poco experimentados es considerar que la originalidad 
solo puede significar realizar un trabajo empírico no realizado nunca antes, o registrar por primera vez por escrito una información novedosa de vital importancia, o explorar áreas de conocimiento y plantear problemas que los especialistas jamás examinaron. Resulta claro que, si se llevan a cabo algunas de las tareas antes descritas, el trabajo resultante será sumamente original. Pero no es necesario llegar a esos extremos. Un trabajo de investigación puede ser original por su tema, por su enfoque, por su presentación o por el contexto en el que se plantea. Es original continuar un trabajo original, propio o ajeno. $\mathrm{O}$ mostrar originalidad al someter a prueba las ideas de otro. También lo es realizar una síntesis que no se llevó a cabo anteriormente, o interpretar de manera novedosa un material muy conocido. Incluso resulta original ensayar en un país algo que sólo se experimentó en otros, o utilizar una técnica muy conocida en un área de conocimiento, pero aplicada en otra diferente. En otras palabras, la exigencia de originalidad de la investigación no debe paralizar al investigador, quien debe obrar confiado en que, si realiza un trabajo honesto, siempre habrá algo original en su proyecto. El desafío cuando uno elabora un proyecto de investigación, es reflexionar y poner en evidencia en qué sentido su trabajo puede ser considerado original, y para ello es muy importante tener en cuenta las características específicas del área en el que se propone desarrollar las labores de investigación planeadas" $(2001)^{26}$.

El problema de la originalidad en los trabajos de investigación, constituye un fenómeno de alcance global. Un recuento de trabajos analizados por país y región, en el año 2013-2014, publicado por Turnitin $2016^{27}$, nos muestra el porcentaje de contenido no original por región. El porcentaje de trabajos que contenían más del $50 \%$ de contenido no original (educación secundaria y superior) fue, $11 \%$ en EE.UU., $12 \%$ en Latinoamérica, $7 \%$ en Reino Unido, $11 \%$ en Europa, $10 \%$ en Oceanía, $9 \%$ en África y Oriente Medio, 14\% en Asia Oriental.

En nuestro estudio, utilizamos dos criterios para evaluar la similitud o coincidencias del texto, que el software informó. En primer lugar, revisamos cada grupo de texto con código de color para determinar si estaba entre comillas. Si el texto estaba citado y correctamente citado, se descontaba dicho porcentaje. El segundo criterio fue evaluar cada grupo de color que no estaban entre comillas para determinar si la coincidencia era realmente contenido no original y si no tenía su referencia, de no ser así, se descontaba del porcentaje reportado inicialmente. Estos criterios mostraron que el texto identificado como coincidencia o similitud, no siempre era válido. Es importante mencionar que algunos de los textos identificados con códigos de color correspondían a grupos de palabras comúnmente utilizadas en las tesis de medicina, por ejemplo, las frases: "Para optar al Título de Médico Cirujano", "Factores de Riesgo", "Variables de Estudio", "Asesor de Tesis" entre otros. Algunos segmentos típicos de las Tesis como el índice, agradecimientos, tablas de operacionalizacion de variables, etc. Suelen sistemáticamente aparecer como texto con coincidencia. Esta claro que los académicos que trabajan en medicina y familiarizados con el desarrollo de tesis, descartarían que estas frases y segmentos típicos podrían corresponder sistematicamente a plagio. Según Larkham y Manns, $2002^{28}$, "a menudo es difícil distinguir entre la erudición pobre, o el descuido y la intención deliberada". Otro punto importante es cuanto representan esos párrafos técnicamente copiados en el esfuerzo del volumen de la tesis, y en cuanto influye realmente en las conclusiones del trabajo.

Turnitin se presenta como una alternativa interesante que puede ayudar a identificar malas prácticas académicas y es uno de los software más conocido y utilizado a escala mundial, pero "Esta herramienta no es infalible". La recomendación es utilizar el software para, posteriormente, efectuar una evaluación exhaustiva.

\section{Limitaciones del estudio}

Nuestro estudio, presenta algunas limitaciones. Primero el tamaño de la muestra. Este reporte está dirigido específicamente a estudiantes de medicina humana de la Universidad Ricardo Palma, en un solo periodo de tiempo, por lo que se necesita incorporar estudios en diversos periodos que nos permitan determinar evolución y tendencias en el tiempo.

La limitación más importante es que fue aplicado a tesis ya concluidas y sustentadas, que no tuvieron oportunidad de revisión por Turnitin previo a su presentación final, por lo que este estudio explora una coincidencia inicial ciega para los estudiantes, sin un mínimo de instrucción ni oportunidad de corregir y adecuar sus trabajos, si hubieran contado con esta herramienta. El uso del sistema probablemente tendría un mayor efecto en el desarrollo moral de los estudiantes, en cuanto a integridad académica. 
Un aspecto importante en el proceso de implementación del Software Turnitin, es que este debe ser programado, con un periodo de capacitación y los instructores deben usar un plan de calificación de coincidencias que los estudiantes perciban como justo y fácil de entender. La mayor ventaja del sistema no es usarlo con un fin policial o punitivo, sino como una herramienta educativa para mejorar la integridad y calidad académica, que es el verdadero objetivo de las universidades.

Recomendamos realizar estudios longitudinales, prospectivos que nos permitan determinar factores relacionados a la originalidad en estudiantes de medicina, la escritura y las habilidades de investigación, así como el nivel de integridad académica de profesores y estudiantes y en qué medida los sistemas tecnológicos de detección contribuyen a esto.

Las implicancias de la originalidad en nuestra práctica deben ser de gran importancia, tanto a nivel personal como institucional, para buscar y promover los más altos estándares de calidad y excelencia académica entre el personal y los estudiantes.

Resulta evidente que debemos hacer mayores esfuerzos para explicar y demostrar a los estudiantes como referenciar las fuentes correctamente, y remarcar la importancia de esto en las prácticas académicas y estándares internacionalmente aceptados. Necesitamos incorporar estos contenidos en los cursos, conferencias y seminarios.

Estamos absolutamente convencidos que la mayor ventaja y aplicación de Turnitin, está durante el proceso de elaboración y ejecución del proyecto de tesis y antes de presentar la versión final de la misma. Los estudiantes y los instructores deberían estar familiarizados con el software y obtener su mayor beneficio. Incorporar prácticas educativas de originalidad en el proceso de evaluación de tesis resulta fundamental.

\section{Limitaciones de software}

Algunos temas requieren atención especial, la tendencia del software a identificar el material como plagiado, incluso si no lo es (Noynaert, 1997)29. Quizas el software no siempre es capaz de comprobar todo en la red, por ejemplo, imágenes, traducciones y algunos programas de computadora. Mulcahy y Goodacre (2004) ${ }^{30}$ también destacan que el software no identifica el texto parafraseado. Al usar dicho software no debería asumirse de que todo el mundo es culpable hasta que se pruebe inocente, ya que esto se alejaría del principio universal de justicia.
Otros problemas prácticos, pero no menos importantes son: capacidad de acceso de los usuarios en la universidad, velocidad de internet disponible, continuidad en el tiempo de la licencia, temas éticos y privacidad de los autores.

Las cuestiones éticas de la honestidad de estudiantes universitarios y la integridad personal son temas de gran relevancia y van más allá de solo el uso de software o reportes automatizados. En este estudio, queda claro que el tema más importante en el uso del software Turnitin está relacionado con la interpretación de los reportes y el proceso necesario de capacitación.

Finalmente existen aspectos en las tesis que pueden y deben ser mejorados. Creemos que los índices de similitud o coincidencias, son indicadores más específicos de "contenido no original", y este contribuiría al grado de originalidad de la tesis, ya que para evaluar esta última, se requiere analizar y tomar en cuenta muchas otras variables y parámetros.

Recomendación: uso rutinario por la Facultad de Medicina Humana-Instituto de Investigación en Ciencias Biomédicas y la Universidad Ricardo Palma del software Turnitin, en el proceso de elaboración y revisión de las tesis de Medicina Humana antes de su presentación final, así como de cualquier producto de investigación, previo a su publicación.

\section{CONCLUSIONES}

Este estudio muestra que el software Turnitin es adecuado para reportar el grado de similitud en las tesis de medicina. El índice de similitud final, constituye un indicador de originalidad, y el software puede ser útil para ayudar a detectar los niveles de originalidad y por lo tanto de la integridad académica en el pregrado. El grado de originalidad hallado en las tesis de medicina humana del curso taller de titulación por tesis 2,015 de la Universidad Ricardo Palma es satisfactorio.

Financiamiento: Apoyo económico por la Universidad Ricardo Palma, forma parte del trabajo: evaluación de calidad de Tesis en Medicina Humana.

Conflicto de interés: Los autores declaran no tener conflictos de interés en la publicación de este artículo.

Recibido: 15 de Julio de 2016

Aprobado: 16 de Agosto de 2016 


\section{REFERENCIAS BIBLIOGRÁFICAS}

1. Paravic, T. Burgos, M. Evaluación de calidad de resúmenes de tesis de un programa de magíster en enfermería. Revista Ciencia y Enfermería; 2009. XV (3):55-68.

2. Ordinola-Sierra C, Tello-Chávez V, VargasPérez J, Rivera-Vílchez R, Alfaro-Carballido D. Análisis de las tesis de pregrado de la Facultad de Odontología de una universidad peruana, 2005-2013. KIRU. 2014;11(1): 25-31.

3. Superintendencia Nacional de Educación Superior Universitaria. Resolución de Consejo Directivo No009-2015-SUNEDU/CD: Reglamento del Registro Nacional de Grados y Titulos. Lima, Perú: Superintendencia Nacional de Educación Superior Universitaria. Disponible en: https://www. sunedu.gob.pe/files/normatividad/Reg_Reg_Nacional_GyT_241215.pdf.

4. Whittle, S. R., \& Eaton, D. G. M. Learning about plagiarism using Turnitin detection software. Medical Education. 2008. 42, 528. doi:10.1111/j.13652923.2008.03058.x.

5. McCabe, D. L., Treviño, L. K., \& Butterfield, K. D. Cheating in academic institutions: A decade of research. Ethics \& Behavior. 2001. 11(3), 219-232.

6. Baker, RK.; Thornton, B.; Adams, M. An Evaluation Of The Effectiveness Of Turnitin.Com As A Tool For Reducing Plagiarism In Graduate Student Term Papers. College Teaching Methods \& Styles Journal (CTMS), v. 4, n. 9 , p. 1-4,Aug. 2011. ISSN 2157-880X.

7. Merriam-Webster Online. 2005. Merriam Webster, Inc. [Electronic Version].

8. Koch, K. Cheating in schools, CQ Researcher. 2000. 10(32), 747-57.

9. Wexler Ellen. Your Dissertation Is Almost Done. What's Next? Turnitin: Plagiarism-detection software is catching on in graduate programs; 2015.

10. Lai-Meng L., Li Xuan W., Cing Chai K., Scientific misconduct encountered by APAME journals: an online survey, Malaysian J Pathol 2015; 37(3) $: 213-218$

11. Carnero, A.M., Mayta-Tristan, P., Konda, K.A. et al. Sci Eng Ethics (2016) doi:10.1007/s11948-016-9820-z .

12. Campbell, C. R., Swift, C. O., \& Denton, L. T. Cheating goes hi-tech: Online term paper mills. Journal of Management Education. 2000. 24(6), 726-740.

13.Villanueva, J. F. Las herramientas antiplagio: necesidad y eficacia. Aula Magna 2.0. [Blog]. 2016. Recuperado de: https://cuedespyd.hypotheses. org/1382.

14. Barrett R., Malcolm J. (2006) Embedding plagiarism education in the assessment process. International Journal for Educational Integrity. 2: 38-45.

15. Batane, T. Turning to Turnitin to Fight Plagiarism among University Students. Educational Technology \& Society, 2010, 13 (2), 1-12.

16. Domínguez-Aroca, M.-I. (2012). Lucha contra el plagio desde las bibliotecas universitarias. El Profesional de La Información, 21(5), 498-503. http://doi.org/10.3145/epi.2012.sep.08.
17. Guillermo Alvarez Llera, et al. Tendencia de la matricula femenina en la educación superior. Un cuarto de siglo. El caso de la carrera de medicina. disponible: http://www.ejournal.unam.mx/rfm/no49-4/RFM49406.pdf.

18. Mendoza Requena, D.; Llanos Zavalaga, F. ; Rosas Aguirre, A.; Contreras Ríos, C. y Huayta Zacarías, E. Factores asociados al ingreso a medicina en la Universidad Peruana Cayetano Heredia y sus tendencias durante el periodo 1994-1999.

19. Carroll J. Institutional issues in deterring, detecting and dealing with plagiarism. 2004.

20. Szabo, A. \& Underwood, J. 'Cybercheats: Is Information and Communication Technology Fuelling Academic Dishonesty?', Active Learning in Higher Education; 2004 . 5(2): 180-99.

21. Carroll J. A Handbook for Deterring Plagiarism in Higher Education, Oxford Centre for Staff and Learning Development; 2002. 96pp.

22. Mccabe, D. L. New Research on Academic Integrity: The Success of "Modified" Honor Codes';2001. Synfax Weekly Reports, http://www.collegepubs.com/ ref/SFX000515.shtml.

23. Frey, C. 'Chips and Cheating: Teachers Are Looking Out for Tech Assisted Dishonesty, even Using Software to Detect Plagiarism, Los Angeles Times 30 August: T1.

24. Nonis, S., \& Swift, C. O. An examination of the relationship between academic dishonesty and workplace dishonesty: A multicampus investigation. Journal of Education for Business, 2001. 76(1), 69-77.

25. Sims, R. L. The relationship between academic dishonesty and unethical business practices. Journal of Education for Business;1993. 668(4), 207-211.

26. Estelle M. Phillips, Derek S. Pugh. obtaining a Ph.D. PUBLICAC. BarceIona Gedisa; 2001. (83-85).

27. Turnitin. Libro Blanco: Integridad Académica en un mundo digital : Índice global de plagio en la educación secundaria y superior Introducción. 2013-2014.

28. Larkham, P. J., \& Manns, S.. Plagiarism and its treatment in higher education. Journal of Further and Higher Education, 2002. 26, 339-349.

29. Noynaert, J. E. Plagiarism detection software;1997. http://www. micsymposium.org/mics_2005/papers/paper97.pdf.

30 Mulcahy, S., \& Goodacre, C. Opening Pandora's box of academic integrity: Using plagiarism detection software; 2004. Proceedings from ASClLITE Conference 2004, Perth, WA. 